\title{
Attrition of the cardiothoracic surgeon-scientist: Definition of the problem and remedial strategies
}

John S. Ikonomidis, MD, PhD, ${ }^{a}$ Philippe Menasché, MD, PhD, ${ }^{b}$ Daniel Kreisel, MD, Frank W. Sellke, MD, ${ }^{\mathrm{d}}$ Y. Joseph Woo, MD, ${ }^{\mathrm{e}}$ and Yolonda L. Colson, $\mathrm{MD}^{\mathrm{f}}$

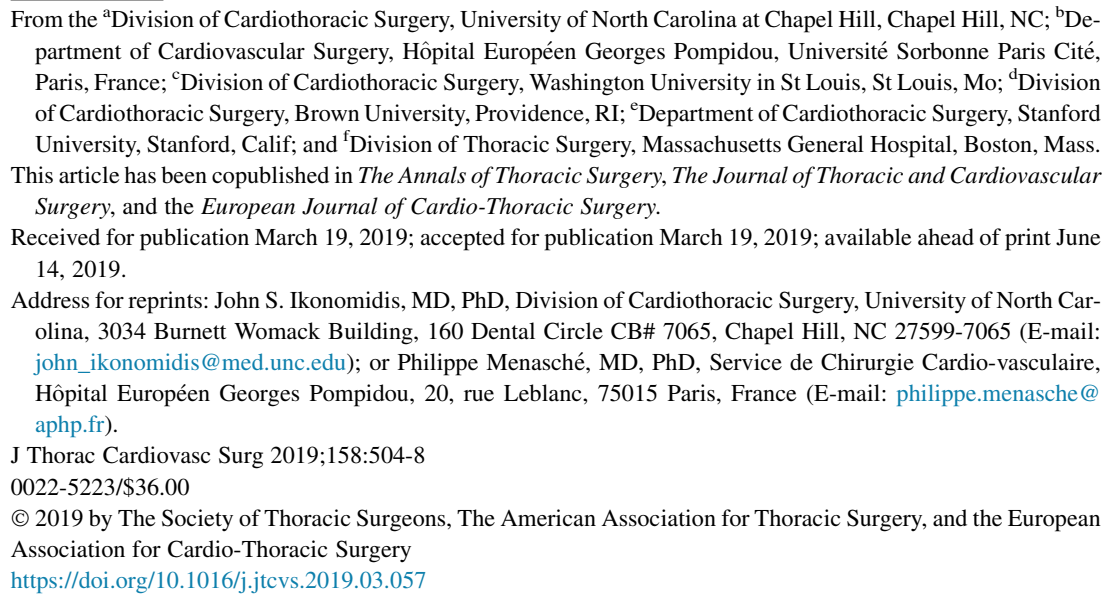

From the ${ }^{\mathrm{a}}$ Division of Cardiothoracic Surgery, University of North Carolina at Chapel Hill, Chapel Hill, NC; ${ }^{\mathrm{b}} \mathrm{De}-$ partment of Cardiovascular Surgery, Hôpital Européen Georges Pompidou, Université Sorbonne Paris Cité, Paris, France; ${ }^{c}$ Division of Cardiothoracic Surgery, Washington University in St Louis, St Louis, Mo; ${ }^{\mathrm{d} D i v i s i o n}$ of Cardiothoracic Surgery, Brown University, Providence, RI; ${ }^{e}$ Department of Cardiothoracic Surgery, Stanford University, Stanford, Calif; and ${ }^{\mathrm{f}}$ Division of Thoracic Surgery, Massachusetts General Hospital, Boston, Mass.

This article has been copublished in The Annals of Thoracic Surgery, The Journal of Thoracic and Cardiovascular Surgery, and the European Journal of Cardio-Thoracic Surgery.

Received for publication March 19, 2019; accepted for publication March 19, 2019; available ahead of print June 14, 2019.

Address for reprints: John S. Ikonomidis, MD, PhD, Division of Cardiothoracic Surgery, University of North Carolina, 3034 Burnett Womack Building, 160 Dental Circle CB\# 7065, Chapel Hill, NC 27599-7065 (E-mail: john_ikonomidis@med.unc.edu); or Philippe Menasché, MD, PhD, Service de Chirurgie Cardio-vasculaire, Hôpital Européen Georges Pompidou, 20, rue Leblanc, 75015 Paris, France (E-mail: philippe.menasche@ aphp.fr).

J Thorac Cardiovasc Surg 2019;158:504-8

$0022-5223 / \$ 36.00$

(C) 2019 by The Society of Thoracic Surgeons, The American Association for Thoracic Surgery, and the European Association for Cardio-Thoracic Surgery

https://doi.org/10.1016/j.jtcvs.2019.03.057

Many definitions have been suggested over the years to describe the activity of physician-scientists. In the purest form, physician-scientists are individuals actively involved in clinical care of patients who also oversee basic or translational research efforts. Specifically, their investigational endeavors are generally aimed at making discoveries that affect patient care through advances in diagnostic modalities or development of new therapeutic strategies. Although discoveries resulting from research conducted by basic scientists without direct involvement with bedside medicine have resulted in major clinical advances, a clear distinction of physician-scientists is their ability to ask clinically relevant questions in the laboratory with a focus on direct clinical translation. Thus, it is not surprising that physician-scientists have contributed to major advances in medical care over the last few decades. Spending prolonged periods of time dedicated to research away from clinical duties have posed unique challenges to surgeons compared with their colleagues in nonoperative fields. Nevertheless, surgeon-scientists have been instrumental in many breakthroughs that have improved the lives of millions of patients. Examples of areas of investigation where basic insights gained by surgeons have advanced clinical care include transplantation immunology, cancer immunotherapy, cardiovascular diseases, and, more recently, reprogramming of adult cells back to pluripotency, the latter leading in 2012 to a Nobel Prize awarded to Shinya Yamanaka, an orthopedic surgeon by training.

\section{THE PROBLEM}

To this end, many North American and European leaders in cardiothoracic surgery have led productive

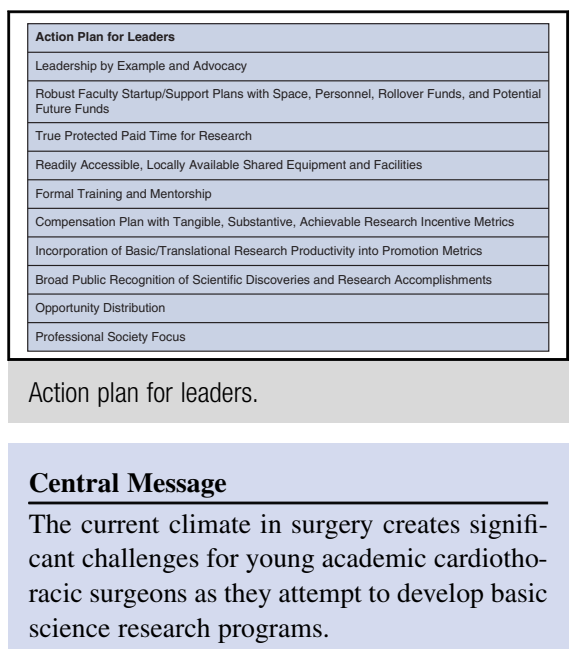

laboratory efforts that have resulted in innovations in the field. Examples of prominent cardiothoracic surgeon-scientists over the last few decades include John Gibbon (the heart-lung machine), ${ }^{1}$ Alfred Blalock and Francis Fontan (surgical procedures for congenital heart defects), 2,3 Norman Shumway (heart transplantation), ${ }^{4}$ Wilfred Bigelow (hypothermia for cardiac surgery), ${ }^{5}$ Melrose and Bentall (hyperkalemic cardiac arrest), ${ }^{6}$ Alain Carpentier (valve bioprostheses), James Cox (antiarrhythmia surgery), ${ }^{8}$ and others. Of note, the research achievements of heart surgeons have occasionally extended beyond their specific area of expertise, as exemplified by the introduction by Clarence Crafoord (in Stockholm) of heparin for prophylaxis of deep venous thrombosis.'

Indeed, research is part of the surgical DNA because it requires curiosity to address unmet medical needs, creativity to handle often unexpected situations, strong commitment to rapidly translate concepts into human therapies, and boldness for implementing them. Conducting exploratory or confirmatory research, executing or supervising experiments, and analyzing their outcomes is one of the most efficient ways of developing critical thinking, which undoubtedly benefits patient care. To this end, a recent editorial in Nature 10 stated that "involvement in research allows surgeons to develop rigor in their everyday work, and to judge the quality of the work done by their 
peers." Likewise, Smythe ${ }^{11}$ cautioned that surgeons who are not interested in "becoming familiar with the terminology and mechanisms behind new therapies run the risk of assuming the role of technicians rather than physicians." He equated such surgeons to "factory workers that have no knowledge of the inner workings of the machines they operate, simply pushing buttons and pulling levers."

However, despite the many rewards that a career as a surgeon-scientist offers, the number of surgeons pursuing such a path has been steadily declining, and a recent editorial in Nature viewed "the flow of surgeons out of research as a problem that must be recognized and stopped" and described the current trend as "dismaying." 10 This decline is particularly distressing if one considers the high number of basic research areas that are directly relevant to clinical problems faced by cardiothoracic surgeons, such as tumor immunology, failure of lung or heart grafts, correction of congenital heart defects, mechanisms of aneurysm formation, and molecular basis of arrhythmias, to name a few.

\section{THE ROOT CAUSES OF THE PROBLEM}

There are several reasons for paucity of cardiothoracic surgeons in basic science research. The first is the high clinical and administrative demands associated with clinical practice. In addition, cardiothoracic surgery is undergoing profound changes that require the acquisition of new skills and training in new technologies such as percutaneous valves or minimally invasive videoassisted procedures. These learning curves are obviously time-consuming and may not leave enough time for conducting research in an increasingly competitive funding environment. At the same time, administrative requirements for documentation and compliance are increasing, and given that cardiothoracic surgery is often a major source of hospital income, administrators tend to push surgeons to increase their caseload rather than spend time in the laboratory. The impact of the surgical specialties on hospital economics is highlighted by the fact that although surgeons represent only $11 \%$ of the physician faculty, their clinical productivity accounts for $33 \%$ of all admissions and $29 \%$ of total revenue generated by all clinical departments. ${ }^{12}$ The pressure to be clinically productive and generate revenue is reported by nearly all academic faculty, and given the time required to become and remain competitive in the marketplace, it is not unexpected that $86 \%$ of surgeons report that this pressure for clinical productivity significantly affects research performance. ${ }^{13}$

A second primary reason cited for declining participation in surgical research is the challenging environment of extramural funding, with competition for grant funding having markedly increased in the United States and Europe over time. ${ }^{14}$ The barriers to success are further magnified for surgeon-scientists, who unfortunately serve as the "canaries in the coal mine" for the greater academic medical community. In some ways, surgeons are the victims of their own success, being caught between the increasing clinical demands to keep departments and hospitals financially solvent in the setting of decreasing reimbursements and the increasingly competitive nature of grant funding for ever decreasing dollars and euros. As an example of the significant decline in available research dollars, the National Institutes of Health (NIH) lost $22 \%$ of its funding capacity between 2003 and $2015 .^{15}$ In this context, the relatively small size of the patient populations affected by surgical diseases and the limited number of funded surgeons available to serve on grant review sessions make grant funding for specific surgical questions difficult to achieve, as evidenced by the $19.1 \%$ decrease in total inflation-adjusted NIH funding in surgical disciplines between 2003 and 2013. ${ }^{16}$ In addition, surgeons usually have less time and less expertise in writing grant applications and, consequently, have a lower application success rate than nonsurgeons, ${ }^{12,13,16,17}$ who typically have more experience with grant applications and thus learn the "tips and tricks" that may render applications attractive. This, in turn, initiates a vicious circle whereby failures lead to discouragement and the recognition that time is better compensated by operative work than by performing research. By 2013, the volume of applications from surgical faculty decreased to below 2004 levels despite the fact that grant applications from the 5 top funded nonsurgical departments increased by approximately $20 \%{ }^{16}$ Another serious adverse effect of declining funding for surgical laboratories is a reduction in the number of surgeon-scientists who are available for mentoring, fewer role models for residents and fellows to emulate in their career, and fewer established surgeons who can help junior colleagues bridge the gap between research during residency and their subsequent clinical career.

Third, the lack of departmental or institutional support may further drive surgeons away from research. Many surgical departments and institutions are driven by clinical productivity due to the large revenue stream derived from surgical procedures and the focus on relative value unitbased compensation systems. ${ }^{18,19}$ Dedicated academic time in these environments is often viewed as a detractor from clinical duties rather than a contribution to the broad academic mission of the department, particularly when grant incomes are not credited to the bottom line of the specific division or department. Furthermore, the dedicated research time required to produce the quality of 
research required for NIH or European funding can be a source of friction among partners because they are required to support a less clinically productive surgeon to work on a research project that may take years to mature and receive funding.

Finally, surgeons who have conducted a research program while keeping a regular surgical practice know that this is achieved by countless "off-hours" work on nights and weekends, well beyond the "normal" working hours. Historically, although surgeon scientists have accepted the attendant sacrifices, this may not be acceptable for the current generation who aspires to a better work-life balance and no longer considers academic advancement and tenure as key drivers of a career. Put together, these observations highlight the concerning reality that although $99 \%$ of surgeon-scientists conducted research activities during residency training, 61\% ended their formal research between the ages of 39 and 49 years, citing an increase in clinical and administrative duties as the most common reasons ( $40 \%$ and $38 \%$, respectively). ${ }^{18}$

\section{POSSIBLE SOLUTIONS FOR KEEPING A SURGEON-SCIENTIST ACTIVITY}

Despite this decline, there are solutions for bringing back academic cardiothoracic surgeons to the field of research. The first requirement is that department chairs, division chiefs, and section heads lead by example (Table 1). They should create the culture and environment that recognize the prominence and value of academic productivity and thus form the foundation upon which faculty members may build highly successful scientific careers. When faculty and trainees observe the importance placed by the leaders in their own research, despite their clinical, educational, and administrative demands, the value of investigation becomes strongly reinforced.

Second, leaders must commit substantive support for new faculty to begin their basic and translational research

\section{TABLE 1. Action plan for leaders}

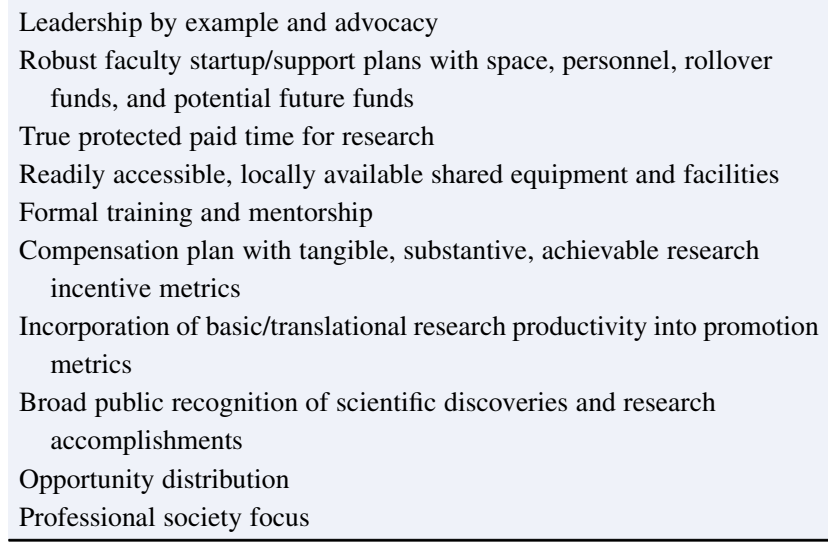

(Table 1). Allocation of laboratory space, ideally in a location close enough to the clinical facilities to prevent distance and travel time from being a disincentive, personnel support in the form of a dedicated research technician, and roll-over funds for supplies are essential. However, this "start-up money" usually will be exhausted after a few years, and thus team leaders need to encourage the younger surgeon-scientists to develop high-quality focused preliminary data and publish high-impact articles that lay the foundations for the arduous process of writing grant applications to secure independent funding. It is beyond the scope of this editorial to detail all the possible funding sources available in Europe and the United States, but there are unique challenges for clinically busy surgeons associated with many of these funding mechanisms. For example, in the United States, the KO8 grant is a potential source for early career funding, but requires at least $75 \%$ of the investigator's time be devoted to research. This is often logistically difficult and prevents the building of a viable clinical practice for a young surgeon. Some have suggested that R21 grants may be better suited, particularly for more senior investigators, with the caveat that this grant mechanism only provides approximately $\$ 150,000$ per year for a maximum of 2 years and thus may not provide enough support and time to generate future funding. Last, some surgical investigators have been successful at leveraging the multiple principle investigator mechanism to achieve R01 funding in collaboration with other surgical and nonsurgical investigators. Likewise, surgeons in Europe can apply for country-specific calls or choose to partner with colleagues from academia and industry to set up more ambitious multidisciplinary projects to be granted by the European Commission.

A third important requirement is to provide the surgeon-scientist true protected time (Table 1). Specifically, this means that time to conduct research cannot occur only between cases, at night, and on weekends. If research is viewed as something to be done "on the side," the demands of clinical practice will prevail every time. Here, there are several approaches that can be helpful. Depending on the local organizations, the modalities of time sharing can vary, and although some may favor alternating practice, that is, 1 week in the clinics and the next one in the laboratory, others will prefer to conduct both activities in parallel to better secure the permanent link between surgical work and research tasks. Inclusion of research time within "normal" working hours serves to reassure young surgeons regarding the preservation of their lives outside of the hospital. However, it should be stressed that to be successful, this approach requires both support and mentorship from the department chairman to unload some teaching and administrative tasks from the surgeon-scientist in favor of specific research activities, as well as an agreement with the other 
clinical partners to carry additional clinical load and perhaps help cover the surgeon-scientist's patients when $\mathrm{he} / \mathrm{she}$ is in the laboratory. Clearly, it is not realistic to oversee laboratory experiments and perform an emergency coronary artery bypass grafting at the same time. However, both types of activities can be reconciled if things are done in an efficient collaborative manner and without procrastination. Although it is difficult to develop a clinical practice and a productive research career while maintaining work-life balance, there are numerous examples of surgeons who maintain a busy surgical practice, receive research grants, review many articles, and travel to present their findings at national and international meetings. It can be done, but it requires both support and dedication.

Furthermore, the recognition that the primary task of a cardiothoracic surgeon is to practice cardiothoracic surgery implies that the commitment of surgeons to perform research should be backed by a strong scientific environment through access to shared equipment and divisional/ departmental core facilities and personnel, as well as robust training in research methodologies and scientific mentorship by basic scientists and other surgeons (Table 1). Further success can be achieved through collaborations of surgeons and basic scientists whereby the scientist provides his/her knowledge of the fundamental knowledge in the field to the clinical understanding provided by the surgeon. Together, they shape the experimental design and interpret data that can then be leveraged by the surgeon as a clinical diagnostic or therapeutic tool.

An additional means for motivating surgeons to participate in surgical research is the development and implementation of a plan that incentivizes scholarly productivity so that increased research productivity based on standard metrics (publications in high-impact journals, patents, extramural funding) should yield enhanced compensation for the research group, the department, and the hospital, depending on the local heath economic system (Table 1). As an example, in France, the government annually gives academic hospitals an amount of money to compensate for the time devoted by physicians to activities not directly related to patient care; this budget aims at offsetting the expenses related to teaching, research, and innovation (ie, charges for research staff and imaging and laboratory facilities) and is modulated according to a series of indicators integrating a publication score $(60 \%)$, the success rate of grant applications, the number of clinical trials, and enrolled patients. Such a system can have a significant impact. In 2017, the amount of money allocated to the Greater Paris academic hospitals within this framework amounted to approximately 600 million euros.

Finally, basic and translational research will only be perceived to be a critical element of our specialty if surgeon-scientists are appointed to leadership positions within cardiothoracic surgery divisions and departments. For this reason, the academic productivity of a faculty member should be taken into consideration when designing departmental promotion metrics, making appointments, and assigning leadership roles within the organization to enhance recognition of basic science research achievements (Table 1). Such a management strategy should be a strong incentive for boosting joint surgery and research pathways.

Assuming that intellectual rigor and reason are intrinsic to the surgical mindset, cardiothoracic surgeons are in an excellent position, provided we are surrounded by experts in methodology and tightly collaborate with basic scientists, to implement experimental protocols, identify and validate clinically relevant targets, and critically analyze outcomes. Leaders must provide support for scientific investigation by setting the example, building culture, and broadly supporting, recognizing, and rewarding research at all levels. Only via relentless pursuit of excellence in these avenues will basic and translational research remain viable and continue to be an essential underpinning of the specialty of cardiothoracic surgery. Central to this goal is the recognition that the qualities required to successfully conduct highquality sustainable research and the outcomes that result from surgeon-initiated research are the most efficient means of ensuring that cardiothoracic patients receive the highest level of care possible with unique solutions designed by the surgeons who study and treat the diseases that threaten their quality of life.

\section{Conflict of Interest Statement}

Authors have nothing to disclose with regard to commercial support.

\section{References}

1. Gibbon JH Jr. Application of a mechanical heart and lung apparatus to cardiac surgery. Minn Med. 1954;37:171-85.

2. Taussig HB, Blalock A. The tetralogy of Fallot; diagnosis and indications for operation; the surgical treatment of the tetralogy of Fallot. Surgery. 1947;21: 145.

3. Fontan F, Baudet E. Surgical repair of tricuspid atresia. Thorax. 1971;26:240-8.

4. Hurley EJ, Dong E Jr, Stofer RC, Shumway NE. Isotopic replacement of the totally excised canine heart. J Surg Res. 1962;2:90-4.

5. Bigelow WG, Callaghan JC, Hopps JA. General hypothermia for experimental intracardiac surgery; the use of electrophrenic respirations, an artificial pacemaker for cardiac standstill, and radio-frequency rewarming in general hypothermia. Trans Meet Am Surg Assoc Am Surg Assoc. 1950;68:211-9.

6. Melrose DG, Dreyer B, Bentall HH, Baker JB. Elective cardiac arrest. Lancet. 1955;269:21-2.

7. Carpentier A. Utilisation d'hétérogreffes dans le traitement des lésions de I'ap pareil valvulaire aortique. Etude biologique et technique. Premiers résultats cliniques. Paris, France: Thèse Med; 1966.

8. Cox JL, Schuessler RB, Cain ME, Corr PB, Stone CM, D'Agostino HJ Jr, et al. Surgery for atrial fibrillation. Semin Thorac Cardiovasc Surg. 1989;1:67-73.

9. Crafoord C, Galie WE. Heparin in surgery. Lancet. 1947;2:482.

10. More surgeons must start doing basic science. Nature. 2017;544:393-4.

11. Smythe WR. The future of academic surgery. Acad Med. 2010;85:768-74.

12. Rangel SJ, Moss RL. Recent trends in the funding and utilization of NIH career development awards by surgical faculty. Surgery. 2004;136:232-9. 
13. Keswani SG, Moles CM, Morowitz M, Zeh H, Kuo JS, Levine MH, et al. Basic science committee of the Society of University Surgeons. The future of basic science in academic surgery: identifying barriers to success for surgeon-scientists. Ann Surg. 2017;265:1053-9.

14. Goldstein AM, Blair AB, Keswani SG, Gosain A, Morowitz M, Kuo JS, et al. A roadmap for aspiring surgeon-scientists in today's healthcare environment. Ann Surg. 2019;269:66-72.

15. NIH Research Funding Trends. Available at: http://faseb.org/Science-PolicyAdvocacy-and-Communications/Federal-Funding-Data/NIH-Research-Funding -Trends.aspx. Accessed December 17, 2018.
16. Hu Y, Edwards BL, Brooks KD, Newhook TE, Slingluff CL Jr. Recent trends in National Institutes of Health funding for surgery: 2003 to 2013. Am J Surg. 2015; 209:1083-9.

17. Mann M, Tendulkar A, Birger N, Howard C, Ratcliffe MB. National Institutes of Health funding for surgical research. Ann Surg. 2008;247:217-21.

18. Zelenock GB, Stanley JC, More RA, Greenfield LJ, Shanley CJ, Jacobs LA. Differential clinical workloads among faculty at a major academic health center. Ann Surg. 1997;226:336-47.

19. Bunton SA, Corrice AM. Trends in tenure for clinical M.D. faculty in U.S. medical schools: a 25-year review. Anal Brief. 2010;9:1-2. 